\title{
State Explosion in Almost-Sure Probabilistic Reachability
}

\author{
François Laroussinie \\ Lab. Spécification \& Vérification, ENS de Cachan \& CNRS UMR 8643, \\ 61, av. Pdt. Wilson, 94235 Cachan Cedex France \\ Jeremy Sproston \\ Dipartimento di Informatica, Università di Torino, 10149 Torino, Italy
}

\begin{abstract}
We show that the problem of reaching a state set with probability 1 in probabilisticnondeterministic systems operating in parallel is EXPTIME-complete. We then show that this probabilistic reachability problem is EXPTIME-complete also for probabilistic timed automata.
\end{abstract}

Key words: probabilistic systems, model checking, computational complexity, formal methods, timed automata

\section{Introduction}

Model checking is an automatic method for guaranteeing that a mathematical model of a system satisfies a formula representing a desired property [4]. Many real-life systems, such as multimedia equipment, communication protocols, networks and fault-tolerant systems, exhibit probabilistic behavior, leading to the study of probabilistic model checking of probabilistic and stochastic models (for an overview, see [12]). We often incorporate nondeterministic choice in probabilistic models, resulting in formalisms akin to Markov decision processes [15]. Furthermore, formalisms such as probabilistic timed automata [11,9] (an

Email addresses: fl@lsv.ens-cachan.fr (François Laroussinie), sproston@di.unito.it (Jeremy Sproston).

1 Supported in part by Miur project Firb-Perf and EEC project Crutial. 
extension of Markov decision processes with clock variables, as in timed automata [2]) can represent models in which nondeterminism, probability and timing information coexist.

The description of a probabilistic system is usually given in terms of interacting sub-systems composed in parallel, or by models referring to variables; an example is the system description language of the probabilistic model-checking tool PRISM [8]. However, the number of system states is exponential in the size of such a description: this is known as the state-explosion problem, and is the main practical limitation of model checking. In this paper, we show that the problem "does there exist a way of resolving the nondeterministic choice of the system such that a set of states is reached with probability 1?" is EXPTIMEcomplete both for a set of probabilistic systems operating in parallel and for probabilistic timed automata. A positive answer to this almost-sure (or qualitative) probabilistic reachability problem establishes that the probabilistic system can guarantee an event (such as the completion of a task) with probability 1. The reachability problem is a fundamental sub-problem of model checking, and, analogously, the almost-sure probabilistic reachability problem is a fundamental sub-problem of probabilistic model checking. Hence, the EXPTIME lower bounds shown in this paper apply to all probabilistic model-checking problems for the systems we consider.

A similar result has been shown by Littman [14] in the context of probabilistic propositional planning, which involves the solution of a probabilistic reachability problem on a concisely-described Markov decision process. Littman's result relies on the reduction of the two-player game $G_{4}$ to reachability on a Markov decision process described in the sequential-effect trees notation. Our approach is instead to reduce the acceptance problem on linearly-bounded alternating Turing machines to the almost-sure probabilistic reachability problem, both on probabilistic systems operating in parallel and on probabilistic timed automata, in a similar manner to the reductions in $[13,1]$.

Preliminaries. An Alternating Turing Machine (ATM) [3] is a tuple $\mathcal{A}=$ $\left(Q, Q_{\vee}, Q_{\wedge}, \Gamma, \delta, q_{0}, q_{a c c}\right)$, with a set $Q=Q_{\vee} \cup Q_{\wedge}$ of states partitioned into disjunctive states $Q_{\vee}$ and conjunctive states $Q_{\wedge}$, an initial state $q_{0} \in Q$, an accepting state $q_{a c c} \in Q_{\vee}$, a tape alphabet $\Gamma=\{a, b\}$, and a transition relation $\delta \subseteq Q \times \Gamma \times Q \times \Gamma \times\{-1,1\}$. A configuration of $\mathcal{A}$ is a triple $\alpha=(q, i, w)$ where $q \in Q$ is the current state, $w \in \Gamma^{*}$ is a word describing the tape content, and $0<i \leq|w|$ is the position of the head on the tape. The symbol written in the $i$ th cell of the tape is denoted by $w(i)$. An ATM moves like a usual nondeterministic Turing machine: for example, if $\alpha=(q, i, w), w(i)=a$ and $\left(q, a, q^{\prime}, b, \varepsilon\right) \in \delta$, then $\mathcal{A}$ may move from $\alpha$ to $\alpha^{\prime}=\left(q^{\prime}, i^{\prime}, w^{\prime}\right)$, where $w^{\prime}$ is $w$ updated by writing $b$ in position $i$, and $i^{\prime}$ is $i+\varepsilon$ (with $i+\varepsilon>0$ ). We say that $\alpha^{\prime}$ is a successor of $\alpha$. We also assume that $\mathcal{A}$ has only one reachable configuration $(q, i, w)$ for which $q=q_{a c c}$, and that $i=1$ and $w=a^{n}$. 
A run of $\mathcal{A}$ from some configuration $\alpha_{0}$ is a tree, the root of which corresponds to $\alpha_{0}$, and where every node corresponding to $\alpha$ has a child node for each successor $\alpha^{\prime}$ of $\alpha$. For $k \in \mathbb{N}$, a run rooted at some disjunctive configuration $\alpha$ is accepting in $k$ steps if and only if its state is $q_{a c c}$ or $k \geq 1$ and at least one of its children is accepting in $k-1$ steps. A run rooted at some conjunctive configuration $\alpha$ is accepting in $k$ steps if and only if $k \geq 1$ and all of its children is accepting in $k-1$ steps (and there is at least one child). A word $v$ is accepted by $\mathcal{A}$ if and only if there exists some $k$ such that the run from $\left(q_{0}, 1, v\right)$ is accepting in $k$ steps. We say that $\mathcal{A}$ is linearly-bounded (LB-ATM) on $v$ if all configurations $(q, i, w)$ in the run of $\mathcal{A}$ have $|w| \leq|v|$. The problem of acceptance of a LB-ATM, which we denote by LB-ATM-ACCEPT, is written as:

Input An ATM $\mathcal{A}$ and a word $v \in \Gamma^{*}$ such that $\mathcal{A}$ is linearly-bounded on $v$. Output YES if and only if $\mathcal{A}$ accepts $v$, No otherwise.

A classical result says that the problem LB-ATM-ACCEPT is EXPTIMEcomplete [3]. In the following, we assume, as in [5], that along a single branch of a run of an LB-ATM, no configuration is repeated; thus every branch is finite. This assumption does not change the complexity issues: one can easily reduce an instance $(\mathcal{A}, v)$ of LB-ATM-ACCEPT to some instance $\left(\mathcal{A}^{\prime}, v^{\prime}\right)$ where $\mathcal{A}^{\prime}$ avoids repetitions by inserting on the tape a counter (encoded in binary) whose value is bounded by $2^{|v|} \cdot|Q| \cdot|v|$ (the maximum number of distinct configurations along the run). Then $\mathcal{A}^{\prime}$ simulates the moves of $\mathcal{A}$ and increases the counter by 1 for every simulated move of $\mathcal{A}$.

\section{Concurrent Markov Decision Processes}

A (discrete) probability distribution over a countable set $Q$ is a function $\mu$ : $Q \rightarrow[0,1]$ such that $\sum_{q \in Q} \mu(q)=1$. For a possibly uncountable set $Q^{\prime}$, let $\operatorname{Dist}\left(Q^{\prime}\right)$ be the set of distributions over countable subsets of $Q^{\prime}$. A distribution $\mu$ will occasionally be denoted by $\{q \mapsto \mu(q) \mid q \in Q$ and $\mu(q)>0\}$. Given the distributions $\mu_{1}, \ldots, \mu_{k}$ over the sets $Q_{1}, \ldots, Q_{k}$, respectively, the independent product $\mu_{1} \otimes \ldots \otimes \mu_{k}$ is defined as $\left\{\left(q_{1}, \ldots, q_{k}\right) \mapsto \mu_{1}\left(q_{1}\right) \cdot \ldots \cdot \mu_{k}\left(q_{k}\right) \mid\left(q_{1}, \ldots, q_{k}\right) \in\right.$ $\left.Q_{1} \times \ldots \times Q_{k}\right\}$. A Markov decision process (MDP) $\mathrm{M}=(\Sigma, S, D)$ comprises a set $\Sigma$ of actions, a set $S$ of states, and the transition relation $D \subseteq S \times \Sigma \times \operatorname{Dist}(S)$. The transitions from state to state of an MDP are performed in two steps: given that the current state is $s$, the first step concerns a nondeterministic selection of an triple $(s, a, \mu) \in D$ associated with $s$; the second step comprises a probabilistic choice, made according to the distribution $\mu$ of the chosen triple, as to which state to make the transition (that is, we move to a state $s^{\prime} \in S$ with probability $\left.\mu\left(s^{\prime}\right)\right)$. We often write $s \stackrel{a}{\rightarrow}_{\mu}$ instead of $(s, a, \mu) \in D$; when $\mu=\left\{s^{\prime} \mapsto 1\right\}$, we write $s \stackrel{a}{\rightarrow} s^{\prime}$. An MDP is finite if $\Sigma, S$ and $D$ are finite sets. 
Unless stated otherwise, we henceforth assume that MDPs are finite.

A finite path is a finite sequence $s_{0}{\stackrel{a_{0}}{\longrightarrow}}_{\mu_{0}} s_{1}{\stackrel{a_{1}}{\longrightarrow}}_{\mu_{1}} \cdots \stackrel{a_{n-1}}{\longrightarrow} \mu_{n-1} s_{n}$ of consecutive transitions followed by a state, such that $\mu_{i}\left(s_{i+1}\right)>0$ for all $i<n$. An infinite path is an infinite sequence $s_{0} \stackrel{a_{0}}{\longrightarrow} \mu_{0} s_{1} \stackrel{a_{1}}{\longrightarrow} \mu_{1} \ldots$ of consecutive transitions, such that $\mu_{i}\left(s_{i+1}\right)>0$ for all $i \in \mathbb{N}$. A state $s$ is reached along the path if there exists $i \in \mathbb{N}$ such that $s=s_{i}$. An adversary of an MDP is a partial function mapping finite paths to triples $(s, a, \mu) \in D$, such that $s$ is the state at the end of the path $[7,17]$. In the standard way, we define the probability measure $\operatorname{Prob}_{s}^{A}$ over measurable sets in the set of paths generated by adversary $A$ from state $s[10]$. Given $F \subseteq S$, let $\operatorname{Reach}_{s}^{A}(F)$ be the set of paths generated by $A$ from $s$ along which a state in $F$ is reached. For an $\mathrm{MDP} \mathrm{M}=(\Sigma, S, D)$, an initial state $\bar{s} \in S$, and a set $F \subseteq S$ of final states, the almost-sure reachability problem for MDPs (MDP-ASR) consists in checking the existence of an adversary of $\mathrm{M}$ that assigns probability 1 to reaching $F$ from $\bar{s}$, and can be solved in polynomial time in the size of $\mathrm{M}$, independently of the transition probabilities (see, for example, [6]). Formally, MDP-ASR is written as:

Input An MDP M, an initial state $\bar{s}$, and a set of final states $F$.

Output YES if and only if there exists an adversary $A$ of $\mathrm{M}$ such that $\operatorname{Prob}_{\bar{s}}^{A}\left\{\operatorname{Reach}_{\bar{s}}^{A}(F)\right\}=1$, No otherwise.

A concurrent Markov decision process $(\mathrm{CMDP}) \mathcal{M}=\left(\mathrm{M}_{1}, \ldots, \mathrm{M}_{k}\right)$ is a $k$-tuple of Markov decision processes. The flattening of the concurrent Markov decision process $\mathcal{M}$ is a Markov decision process $(\Sigma, S, D)$, where $\Sigma=\Sigma_{1} \cup \ldots \cup \Sigma_{k}$, $S=S_{1} \times \ldots \times S_{k}$, and $D$ is the set of all triples $\left(\left(s_{1}, \ldots, s_{k}\right), a, \mu\right)$ from $S \times$ $\Sigma \times \operatorname{Dist}(S)$ such that $\mu=\mu_{1} \otimes \ldots \otimes \mu_{k}$, where, for each $1 \leq i \leq k$, either $\left(s_{i}, a, \mu_{i}\right) \in D_{i}$ or $\left(a \notin \Sigma_{i}\right.$ and $\left.\mu_{i}=\left\{s_{i} \mapsto 1\right\}\right)[16]$. For a concurrent Markov decision process $\mathcal{M}=\left(\mathrm{M}_{1}, \ldots, \mathrm{M}_{k}\right)$ with the flattening $\mathrm{M}=(\Sigma, S, D)$, an initial state $\left(\bar{s}_{1}, \ldots, \bar{s}_{k}\right) \in S$ and a set of final states $F \subseteq S$ of $\mathcal{M}$, the almost-sure reachability problem for CMDPs (CMDP-ASR) is similar to MDP-ASR, but checks for the existence of an adversary on the flattening of the CMDP:

Input $\mathrm{A}$ CMDP $\mathcal{M}$, an initial state $\bar{s}$, and a set of final states $F$.

Output YES if and only if there exists an adversary $A$ of the flattening $\mathrm{M}$ of $\mathcal{M}$ such that $\operatorname{Prob}_{\bar{s}}^{A}\left\{\operatorname{Reach}_{\bar{s}}^{A}(F)\right\}=1$, No otherwise.

Theorem 2.1 The problem CMDP-ASR is EXPTIME-complete.

Proof. An EXPTIME algorithm is obtained by applying standard polynomial time algorithms for MDP-ASR [6] over the (exponential) flattening of the CMDP in question. It remains to show the EXPTIME-hardness of CMDPASR. Let $v \in \Gamma^{n}$ and $\mathcal{A}=\left(Q, Q_{\vee}, Q_{\wedge}, \Gamma, \delta, q_{0}, q_{a c c}\right)$ be an LB-ATM. We define a $\mathrm{CMDP} \mathcal{M}_{\mathcal{A}, v}=\left(\mathrm{M}^{\mathrm{cntrl}}, \mathrm{M}_{1}, \ldots, \mathrm{M}_{n}\right)$ which models the run of $\mathcal{A}$ over $v$ :

- For each $1 \leq i \leq n$, the MDP $\mathrm{M}_{i}$ models the $i$ th tape cell. The state set 
is $S_{i}=\left\{s_{a}^{i}, s_{b}^{i}\right\}$, and the initial state is $s_{a}^{i}$ if $v(i)=a$, and $s_{b}^{i}$ otherwise. The alphabet is $\Sigma_{i}=(\delta \times\{i\}) \cup\{(a, i),(b, i)\}$. For each transition $t=$ $\left(q, e, q^{\prime}, e^{\prime}, \varepsilon\right) \in \delta$ such that $i+\varepsilon \in\{1, \ldots, n\}$, there is a transition $s_{e}^{i} \stackrel{t, i}{\longrightarrow} s_{e^{\prime}}^{i}$ in $D_{i}$ to simulate the behavior of $t$. Furthermore, for each $e \in\{a, b\}$, there is a transition $s_{e}^{i} \stackrel{e, i}{\longrightarrow} s_{e}^{i}$ in $D_{i}$ to indicate the current value of the cell.

- The MDP $\mathrm{M}^{\mathrm{cntrl}}=\left(\Sigma^{\mathrm{cntrl}}, S^{\mathrm{cntrl}}, D^{\mathrm{cntrl}}\right)$ models the control part of $\mathcal{A}$. The alphabet is $\Sigma^{\mathrm{cntrl}}=(\delta \times\{1, \ldots, n\}) \cup(\{a, b\} \times\{1, \ldots, n\})$, and the state set is $S^{\mathrm{cntrl}}=(Q \times\{1, \ldots, n\}) \cup\left(Q_{\wedge} \times\{1, \ldots, n\} \times \delta\right)$. The initial state is $\left(q_{0}, 1\right)$. The transition relation $D^{\text {cntrl }}$ is defined as follows:

- For each $q \in Q_{\vee}$, each $1 \leq i \leq n$, and each $t \in \delta$, a transition $(q, i) \stackrel{t, i}{\longrightarrow}$ $\left(q^{\prime}, i+\varepsilon\right)$ is included in $D^{\text {cntrl }}$ if $t=\left(q, e, q^{\prime}, e^{\prime}, \varepsilon\right)$ and $i+\varepsilon \in\{1, \ldots, n\}$.

- For each $q \in Q_{\wedge}$, each $1 \leq i \leq n$ and each $e \in\{a, b\}$ such that the set $T_{q, i, e}=\left\{\left(q, e, q^{\prime}, e^{\prime}, \varepsilon\right) \in \delta \mid i+\varepsilon \in\{1, \ldots, n\}\right\}$ is non empty, we have a transition $(q, i) \stackrel{e, i}{\rightarrow} \mu_{(q, i, e)}$ in $D^{\text {cntrl }}$, where $\mu_{(q, i, e)}$ is the distribution (with equal probabilities) over the states $(q, i, t)$ for all $t \in T_{q, i, e}$. Then we add transitions $(q, i, t) \stackrel{t, i}{\longrightarrow}\left(q^{\prime}, i+\varepsilon\right)$ to $D^{\text {cntrl }}$ according to the definition of $t$.

The size of $\mathcal{M}_{\mathcal{A}, v}$ is $O(n \times|Q| \times|\delta|)$, including the probabilities represented as the ratio of two integers encoded in binary, and the reduction can be done in logarithmic space. Now we show that $\mathcal{A}$ accepts $v$ if and only if CMDP-ASR returns YES for $\mathcal{M}_{\mathcal{A}, v}$ with the initial state $\left(\left(q_{0}, 1\right), s_{v(1)}^{1}, \ldots, s_{v(n)}^{n}\right)$, and the set containing the single final state $\left(\left(q_{a c c}, 1\right), s_{a}^{1}, \ldots, s_{a}^{n}\right)$. As the problem LB-ATMACCEPT is EXPTIME-hard, this will suffice to show the EXPTIME-hardness of CMDP-ASR.

In the following, for a given word $w \in \Gamma^{n}$, we write $\mathbf{s}_{w}$ instead of $s_{w(1)}^{1}, \ldots, s_{w(n)}^{n}$. Let $\mathrm{M}_{\mathcal{A}, v}=(\Sigma, S, D)$ be the flattening of the CMDP $\mathcal{M}_{\mathcal{A}, v}$. Our first task is to construct a modified, action-less version of $\mathrm{M}_{\mathcal{A}, v}$, denoted by $\overline{\mathrm{M}}=(\bar{S}, \bar{D})$, so that we are better able to relate the transitions of $\mathcal{M}_{\mathcal{A}, v}$ with those of $\mathcal{A}$. Intuitively, we obtain $\overline{\mathrm{M}}$ by removing intermediate states of the form $\left((q, i, t), \mathbf{s}_{w}\right)$ from $\mathrm{M}_{\mathcal{A}, v}$. Let $\bar{S}_{N} \subseteq S$ be the set of states of $\mathcal{M}_{\mathcal{A}, v}$ which have the component $\mathrm{M}^{\text {cntrl }}$ in a state in the set $Q_{\vee} \times\{1, \ldots, n\}$, and similarly let $\bar{S}_{P} \subseteq S$ be the set of states for which $\mathrm{M}^{\mathrm{cntrl}}$ is in a state in $Q_{\wedge} \times\{1, \ldots, n\}$. Then let $\bar{S}=\bar{S}_{N} \cup \bar{S}_{P}$. The transition relation $\bar{D} \subseteq \bar{S} \times \operatorname{Dist}(\bar{S})$ is defined as follows. For states $\left((q, i), \mathbf{s}_{w}\right) \in \bar{S}_{N}$, for each transition $\left((q, i), \mathbf{s}_{w}\right) \stackrel{t, i}{\longrightarrow}\left(\left(q^{\prime}, i^{\prime}\right), \mathbf{s}_{w^{\prime}}\right)$ of $D$ we have $\left((q, i), \mathbf{s}_{w}\right) \rightarrow\left(\left(q^{\prime}, i^{\prime}\right), \mathbf{s}_{w^{\prime}}\right)$ in $\bar{D}$. For states $\left((q, i), \mathbf{s}_{w}\right) \in \bar{S}_{P}$, observe that in $\mathbf{M}_{\mathcal{A}, v}$ we have transitions $\left((q, i), \mathbf{s}_{w}\right) \stackrel{w(i), i}{\longrightarrow} \mu\left((q, i, t), \mathbf{s}_{w}\right)$, and, from $\left((q, i, t), \mathbf{s}_{w}\right)$, there is a unique transition $\left((q, i, t), \mathbf{s}_{w}\right) \stackrel{t, i}{\longrightarrow}\left(\left(q^{\prime}, i^{\prime}\right), \mathbf{s}_{w^{\prime}}\right)$, where $q^{\prime}$, $i^{\prime}$ and $w^{\prime}$ depend on $t$. In $\overline{\mathrm{M}}$ we skip the intermediate state $\left((q, i, t), \mathbf{s}_{w}\right)$ and consider a transition $\left((q, i), \mathbf{s}_{w}\right) \rightarrow_{\bar{\mu}}$ such that $\bar{\mu}\left(\left(q^{\prime}, i^{\prime}\right), \mathbf{s}_{w^{\prime}}\right)$ equals $\mu\left((q, i, t), \mathbf{s}_{w}\right)$ if there is a transition $t$ such that $\left((q, i, t), \mathbf{s}_{w}\right) \rightarrow\left(\left(q^{\prime}, i^{\prime}\right), \mathbf{s}_{w^{\prime}}\right)$, and 0 otherwise. We can verify that, for all states $s \in \bar{S}$ and any $F \subseteq \bar{S}$, CMDP-ASR returns YES for $\mathcal{M}_{\mathcal{A}, v}, s$ and $F$ if and only if MDP-ASR returns YES for $\overline{\mathrm{M}}, s$ and $F$. 
Note that we can obtain an isomorphism between the configurations of $\mathcal{A}$ and the states of $\overline{\mathrm{M}}$, which relates a configuration $(q, i, w)$ of $\mathcal{A}$ to a state $\left((q, i), \mathbf{s}_{w}\right)$ of $\overline{\mathrm{M}}$. For configurations $(q, i, w)$ and $\left(q^{\prime}, i^{\prime}, w^{\prime}\right)$, we have that $\left(q^{\prime}, i^{\prime}, w^{\prime}\right)$ is a successor of $(q, i, w)$ if and only if $\left((q, i), \mathbf{s}_{w}\right) \rightarrow_{\mu}\left(\left(q^{\prime}, i^{\prime}\right), \mathbf{s}_{w^{\prime}}\right)$. Because no configuration is repeated along a branch of a run of $\mathcal{A}$ (see page 3), the MDP $\overline{\mathrm{M}}$ is acyclic (i.e. there does not exist a finite path $s_{0}{\stackrel{a_{0}}{\longrightarrow}}_{\mu_{0}} s_{1}{\stackrel{a_{1}}{\longrightarrow}}_{\mu_{1}} \cdots \stackrel{a_{n-1}}{\longrightarrow} \mu_{n-1} s_{n}$ of $\mathrm{M}$ such that $s_{0}=s_{n}$ ). Hence $\overline{\mathrm{M}}$ has no infinite path.

Next, we introduce the alternating reachability problem $(A L T-R E A C H)$ on $\overline{\mathrm{M}}$. First we consider the variant in $k$ steps $(A L T-R E A C H-k)$ :

Input An MDP M, a partition of the states of M into disjunctive states $S_{\vee}$ and conjunctive states $S_{\wedge}$, an initial state $s$, and a set of final states $F$.

Output YES if and only if:

- $s \in S_{\vee}$ and either $s \in F$ or $k \geq 1$ and there exists a transition $s \rightarrow_{\mu} s^{\prime}$ such that ALT-REACH- $(k-1)$ returns YES on M, $S_{\vee}, S_{\wedge}, s^{\prime}$, and $F$;

- $s \in S_{\wedge}, k \geq 1$, and, for all states $s^{\prime} \in S$, we have that $s \rightarrow_{\mu} s^{\prime}$ implies that ALT-REACH- $(k-1)$ returns YES on M, $S_{\vee}, S_{\wedge}, s^{\prime}$, and $F$;

and No otherwise.

Then the answer to ALT-REACH is YES if and only if there exists some $k$ such that the corresponding instance of ALT-REACH- $k$ is positive. We apply the problem ALT-REACH by letting the set of disjunctive and conjunctive states considered be equal to $\bar{S}_{N}$ and $\bar{S}_{P}$, respectively. From the acyclic property of $\overline{\mathrm{M}}$, we have that the problem MDP-ASR outputs YES on $\overline{\mathrm{M}}, s$ and $F$ if and only if ALT-REACH outputs YES on $\overline{\mathrm{M}}, \bar{S}_{N}, \bar{S}_{P}, s$ and $F .{ }^{2}$ We claim that the following statements are equivalent:

(1) CMDP-ASR returns YES on input $\mathcal{M}_{\mathcal{A}, v},\left(\left(q_{0}, 1\right), \mathbf{s}_{v}\right)$, and $\left(\left(q_{a c c}, 1\right), \mathbf{s}_{a \ldots a}\right)$;

(2) MDP-ASR returns YES on input $\overline{\mathrm{M}},\left(\left(q_{0}, 1\right), \mathbf{s}_{v}\right)$, and $\left(\left(q_{a c c}, 1\right), \mathbf{s}_{a \ldots a}\right)$;

(3) ALT-REACH returns Yes on input $\overline{\mathrm{M}},\left(\left(q_{0}, 1\right), \mathbf{s}_{v}\right)$, and $\left(\left(q_{a c c}, 1\right), \mathbf{s}_{a \ldots a}\right)$;

(4) LB-ATM-ACCEPT returns Yes on input $\mathcal{A}$ and $v$.

The equivalence of statements (1) and (2), (statements (2) and (3), respectively) follows from the arguments relating CMDP-ASR and MDP-ASR (MDPASR and ALT-REACH, respectively) given above. The equivalence of statements (3) and (4) follows from the aforementioned isomorphism between configurations of $\mathcal{A}$ and states of $\bar{M}$. Hence, the CMDP-ASR problem and the acceptance problem for LB-ATM are equivalent, and thus the CMDP-ASR problem is EXPTIME-hard.

$\overline{2}$ The proof can be done directly on the number of steps of the accepting runs, which is the same in both problems. 


\section{Probabilistic Timed Automata}

In this section, we study the complexity of the almost-sure probabilistic reachability problem for probabilistic timed automata. We use standard notation from (probabilistic) timed automata, such as clock valuations val $: \mathcal{X} \rightarrow \mathbb{R}_{\geq 0}$ which are mappings from the set of clocks $\mathcal{X}$ to the set of non-negative real numbers $\mathbb{R}_{\geq 0}$, and clock constraints $\Psi_{\mathcal{X}}$ over $\mathcal{X}$. A probabilistic timed automaton (PTA) $\mathrm{P}=(L, \mathcal{X}$, prob $)[11,9]$ is a tuple consisting of a finite set $L$ of locations, a finite set $\mathcal{X}$ of clocks, and a finite set prob $\subseteq L \times \Psi_{\mathcal{X}} \times \operatorname{Dist}\left(2^{\mathcal{X}} \times L\right)$ of probabilistic edges. A probabilistic edge $(l, g, p) \in$ prob is a triple containing (1) a source $l$ location, (2) a guard $g$, and (3) a probability distribution $p$ which assigns probability to pairs of the form $\left(X, l^{\prime}\right)$ for some clock reset $X$ and target location $l^{\prime}$. The semantics of $\mathrm{P}$ is the action-less, infinite-state Markov decision process $\mathrm{M}[\mathrm{P}]=(S, D)$. The state set $S=L \times \mathbb{R}_{\geq 0}^{\mathcal{X}}$ comprises location-valuation pairs. The transition relation $D$ is defined as the smallest set such that $((l, \mathrm{val}), \delta, \mu) \in D$ if there exist $\delta \in \mathbb{R}_{\geq 0}$ and a probabilistic edge $(l, g, p) \in$ prob such that (1) val $+\delta \models g$ and (2) for each $\left(l^{\prime}, v a l^{\prime}\right) \in S$, we have $\mu\left(l^{\prime}, v a l^{\prime}\right)=\sum_{X \subseteq \mathcal{X} \& v a l^{\prime}=(v a l+\delta)[X:=0]} p\left(X, l^{\prime}\right)$.

Let $\mathbf{0} \in \mathbb{R}_{\geq 0}^{\mathcal{X}}$ be the clock valuation which assigns 0 to all clocks in $\mathcal{X}$. For a probabilistic timed automaton $\mathrm{P}=(L, \mathcal{X}$, prob $)$, an initial location $\bar{l} \in L$, and a set $L_{F} \subseteq L$ of final locations, the almost-sure reachability problem for PTAs consists in checking the existence of an adversary that assigns probability 1 to reaching $L_{F}$ from $(\bar{l}, \mathbf{0})$. Formally, PTA-ASR is the problem written as:

Input A PTA $\mathrm{P}$, an initial location $\bar{l}$, and a set of final locations $L_{F}$.

Output YES if and only if there exists an adversary $A$ of $\mathrm{M}[\mathrm{P}]$ such that $\operatorname{Prob}_{(\bar{l}, \mathbf{0})}^{A}\left\{\operatorname{Reach}_{(\bar{l}, \mathbf{0})}^{A}\left(L_{F} \times \mathbb{R}_{\geq 0}^{\mathcal{X}}\right)\right\}=1$, No otherwise.

Kwiatkowska et al. [11] show that the problem PTA-ASR can be solved in exponential time in the size of $\mathrm{P}$ using a variant of the region graph technique for timed automata [2]. We now show that this bound is optimal.

Theorem 3.1 The problem PTA-ASR is EXPTIME-complete.

Proof. Given that an EXPTIME algorithm has been presented previously, it remains to show the EXPTIME-hardness of PTA-ASR. Let $\mathcal{A}=\left(Q, Q_{\vee}, Q_{\wedge}\right.$, $\left.\Gamma, \delta, q_{0}, q_{a c c}\right)$ be an LB-ATM and $v$ be a word of length $n$. We define a PTA $\mathrm{P}_{\mathcal{A}, v}=(L, \mathcal{X}$, prob $)$ which models the run of $\mathcal{A}$ over $v$. Then we let $L=$ $(Q \times\{1, \ldots, n\}) \cup\left\{\bar{l}, l_{F}\right\}$, and $\mathcal{X}=\left\{x_{1}, \ldots, x_{n}, \bar{x}_{1}, \ldots, \bar{x}_{n}, y\right\}$. The contents of the tape of $\mathcal{A}$ are encoded by the relative values of the clocks $x_{1}, \ldots, x_{n}, \bar{x}_{1}, \ldots, \bar{x}_{n}$ : cell $i$ contains $a$ if $x_{i}=\bar{x}_{i}$, and $b$ if $x_{i}<\bar{x}_{i}$. Clock $y$ is used to ensure the elapse of time.

The probabilistic edge relation prob of $\mathrm{P}_{\mathcal{A}, v}$ is obtained in a similar way to 
the transition relation of the CMDP of the proof of Theorem 2.1, as we now explain. The idea is that probabilistic edges emulate the transitions of $\mathcal{A}$ : in particular, the guards of probabilistic edges from a given location $(q, i)$ can test whether the current tape symbol is $a$ or $b$ by checking whether $x_{i}=\bar{x}_{i}$ or $x_{i}<\bar{x}_{i}$, respectively. Furthermore, the writing of a symbol in a tape cell can be replicated by clock resets: for example, to represent the writing of $a$ in cell $i$, we reset clocks $x_{i}$ and $\bar{x}_{i}$ to 0 (so that $x_{i}=\bar{x}_{i}$ ), whereas to write $b$ we reset only $x_{i}$ (so that $x_{i}<\bar{x}_{i}$ ). The target locations of the probabilistic edges are derived from the target states of the transition of $\mathcal{A}$ involved in the definition of the probabilistic edge, and by the associated movement of the tape head.

In a location $(q, i)$ derived from a disjunctive ATM state (that is $q \in Q_{\vee}$ ), there will be a nondeterministic choice between probabilistic edges, each of which is derived from a transition of $\mathcal{A}$ from $q$, and each of which will assign probability 1 to a single outcome. In contrast, in a location $(q, i)$ derived from a conjunctive ATM state (that is $q \in Q_{\wedge}$ ), there are at most two probabilistic edges, one of which has a guard testing whether the current tape symbol is $a$ (using $x_{i}=\bar{x}_{i}$, as above), the other testing for $b$ (using $x_{i}<\bar{x}_{i}$ ). The probabilistic branching is done (with equal probability) over the various outcomes derived from the outgoing transitions of $q$ labeled with $a$ or $b$, respectively. To the guard of each probabilistic edge, we add the constraint $y>0$ to force some time to elapse, in order to ensure that a clock reset of $\left\{x_{j}\right\}$ encodes the writing of $b$ in cell $j$. Finally, we add the probabilistic edge $\left(\bar{l}, y>0,\left\{X_{v},\left(q_{0}, 1\right) \mapsto 1\right\}\right)$, where $X_{v}=\left\{x_{i} \mid v(i)=b\right\} \cup\{y\}$, to encode the initialization of the input word $v$ on the tape, and also the probabilistic edge $\left(\left(q_{a c c}, 1\right), \bigwedge_{i=1}^{n}\left(x_{i}=\bar{x}_{i}\right),\left\{\emptyset, l_{F} \mapsto 1\right\}\right)$.

The size of the PTA $\mathrm{P}_{\mathcal{A}, v}$ is linear in $|\mathcal{A}| \cdot|v|$ : we have $|L|=|Q| \cdot|v|+2$, $|\mathcal{X}|=2 \cdot|v|+1$, and the size of the probabilistic edge set prob-including the probabilities encoded in binary - is bounded by $2 \cdot|v| \cdot|\delta|$. The reduction can be done in logarithmic space. Then $\mathcal{A}$ accepts $v$ if and only if PTA-ASR returns YES on the input PTA $\mathrm{P}_{\mathcal{A}, v}$, the initial location $\bar{l}$, and the set $\left\{l_{F}\right\}$ comprising the single final location $l_{F}$. Hence PTA-ASR is EXPTIME-hard.

\section{References}

[1] L. Aceto and F. Laroussinie. Is your model checker on time? On the complexity of model checking for timed modal logics. Journal of Logic and Algebraic Programming, 52-53:7-51, 2002.

[2] R. Alur and D. L. Dill. A theory of timed automata. Theoretical Computer Science, 126(2):183-235, 1994.

[3] A. K. Chandra, D. Kozen, and L. J. Stockmeyer. Alternation. Journal of the ACM, 28(1):114-133, 1981. 
[4] E. M. Clarke, O. Grumberg, and D. Peled. Model checking. MIT Press, 1999.

[5] C. Courcoubetis and M. Yannakakis. The complexity of probabilistic verification. Journal of the ACM, 42(4):857-907, 1995.

[6] L. de Alfaro. Computing minimum and maximum reachability times in probabilistic systems. In Proc. of the 10th Int. Conf. on Concurrency Theory (CONCUR'99), volume 1664 of $L N C S$, pages 66-81. Springer, 1999.

[7] S. Hart, M. Sharir, and A. Pnueli. Termination of probabilistic concurrent program. ACM Trans. Program. Lang. Syst., 5(3):356-380, 1983.

[8] A. Hinton, M. Kwiatkowska, G. Norman, and D. Parker. PRISM: A tool for automatic verification of probabilistic systems. In Proc. of the 12th Int. Conf. on Tools and Algorithms for the Construction and Analysis of Systems (TACAS'06), volume 3920 of LNCS, pages 441-444. Springer, 2006.

[9] H. E. Jensen. Model checking probabilistic real time systems. In Proc. of the 7th Nordic Workshop on Programming Theory, volume 86, pages 247-261. Chalmers Institute of Technology, 1996.

[10] J. G. Kemeny, J. L. Snell, and A. W. Knapp. Denumerable Markov Chains. Graduate Texts in Mathematics. Springer, 2nd edition, 1976.

[11] M. Kwiatkowska, G. Norman, R. Segala, and J. Sproston. Automatic verification of real-time systems with discrete probability distributions. Theoretical Computer Science, 286:101-150, 2002.

[12] M. Z. Kwiatkowska. Model checking for probability and time: from theory to practice. In Proc. of the 18th IEEE Symposium on Logic in Computer Science (LICS 2003), pages 351-360. IEEE Computer Society, 2003.

[13] F. Laroussinie and Ph. Schnoebelen. The state-explosion problem from trace to bisimulation equivalence. In Proc. of the 3rd Inter. Conf. on Foundations of Software Science and Computation Structures (FoSSaCS 2000), volume 1784 of LNCS, pages 192-207. Springer, 2000.

[14] M. L. Littman. Probabilistic propositional planning: Representations and complexity. In Proc. of the 14th National Conf. on Artificial Intelligence and the 9th Innovative Applications of Artificial Intelligence Conf. (AAAI/IAAI'97), pages 748-754. AAAI Press/MIT Press, 1997.

[15] M. L. Puterman. Markov Decision Processes. J. Wiley \& Sons, 1994.

[16] R. Segala and N. A. Lynch. Probabilistic simulations for probabilistic processes. Nordic Journal of Computing, 2(2):250-273, 1995.

[17] M. Y. Vardi. Automatic verification of probabilistic concurrent finite-state programs. In Proc. of the 16th An. Symp. on Foundations of Computer Science (FOCS'85), pages 327-338. IEEE Computer Society Press, 1985. 